\title{
Racial/Ethnic Differences Among Tumor Infiltrating Lymphocytes in Breast Cancer Tumors
}

\section{Surbhi Bansil}

University of Hawai'i at Manoa John A Burns School of Medicine

\section{Anthony Silva}

University of Hawai'i at Manoa John A Burns School of Medicine

\section{Alana Taniguchi}

HRP: Hawaii Residency Programs Inc

\section{Christina Wiedmer}

HRP: Hawaii Residency Programs Inc

\section{Mayumi Fernandez}

University of Hawai'i at Manoa John A Burns School of Medicine

\section{lan Pagano}

UH Cancer Center: University of Hawai'i Cancer Center

\section{Koah Vierkoetter}

Queen's Medical Center

Jeffrey Killeen

Kapi'olani Medical Center for Women and Children

Jami Fukui ( $\nabla$ jfukui@cc.hawaii.edu )

University of Hawai'i Cancer Center https://orcid.org/0000-0002-0248-266X

\section{Research Article}

Keywords: Breast cancer, race, tumor infiltrating lymphocytes (TILs), tumor microenvironment

Posted Date: September 9th, 2021

DOI: https://doi.org/10.21203/rs.3.rs-874109/v1

License: (c) (1) This work is licensed under a Creative Commons Attribution 4.0 International License.

Read Full License 


\section{Abstract}

\section{Purpose}

Tumor infiltrating lymphocytes (TILs) have emerged as a predictor of cancer treatment response and patient outcomes, including for breast cancer. Current studies investigating racial/ethnic differences in TILs and immune profiles in breast cancer offer varying results. Our study hopes to address the paucity of data in breast cancer tumor microenvironment from different racial/ethnic groups not well represented in the literature.

\section{Methods}

We reviewed 183 cases of women diagnosed with early stage breast cancer who received neoadjuvant treatment at two large health systems in Hawaii between 2008 and 2020. We evaluated clinical and demographic information including: age at diagnosis, race/ethnicity, tumor stage, tumor subtype according to ER, PR, and HER2 receptor status and compared them with obtaining a pathologic complete response (pCR) and amount of stromal TILs (sTILs).

\section{Results}

We found a significantly greater amount of sTILs in Asians $(37.7 \%, p=0.01)$ and Native Hawaiian/Pacific Islander (NHPI) $(37.2 \%, \mathrm{p}=0.02)$ patients compared to White patients on multivariate analysis. We found no significant differences in $\mathrm{pCR}$ among the different racial/ethnic groups.

\section{Conclusions}

Racial/ethnic differences in the amount of sTILs in breast cancer tumors suggest that higher STIL percentages alone do not predict for pCR. Increases in sTILs in Asian and NHPI patients suggest differences in immune cell profiles in the breast tumor microenvironment. This may in part contribute to known outcome disparities in these populations.

\section{Introduction}

The tumor microenvironment, specifically tumor infiltrating lymphocytes (TILs) in breast cancer, have been gaining more attention. TILs incorporate a heterogeneous composition of T-cells, B-cells, and other lymphocytes, and it is an emerging biomarker that is both predictive of cancer treatment response and prognostic of cancer outcomes [1]. Certain breast cancer subtypes-triple negative (TN, ER-/PR-/HER2-) and HER2+-which are known to have higher stromal TIL (STIL) amounts, have also been associated with improved prognosis and response to chemotherapy [2] which can aid clinicians in predicting response to neoadjuvant chemotherapy. Specific TILs, namely CD8+ T cells, are associated with an increase in obtaining a pathologic complete response (pCR) after neoadjuvant therapy, regardless of breast cancer subtype [3]. 
Differences in TILs among various racial/ethnic groups have been evaluated. The Women's Circle of Health Study examined differences in TILs, particularly CD8+ T cells, in breast tumors between Black and White women and found that Black women with TN breast cancer and high CD8+ T cell density were found to have better overall survival than patients with low CD8+ T cell density [4]. Another study in HER2+ breast tumors showed the presence of CD8+ T cells was associated with a decrease in mortality [5]. Chen et al, examined gene expression microarray data of Asian and White patients with breast cancer, and found Asians to have significantly higher TILs than White patients, particularly in patients with breast cancer of luminal B and HER2+ subtypes [6].

We examined sTILs in early stage breast tumors who had received neoadjuvant treatment from two major health systems in Hawaii from 2008-2020. Our study hopes to address the paucity of data in breast cancer tumor microenvironment from different racial/ethnic groups not well represented in the literature.

\section{Methods}

This study evaluated 183 cases of early stage breast cancer who received neoadjuvant treatment from two large health systems from 2008-2020. A retrospective chart review was performed along with pathological evaluation from core biopsies and subsequent surgical excisions. sTILs were determined according to recommendations proposed by the International Immuno-Oncology Biomarker Working Group in 2017, separating the percent of stromal and intratumoral TILs [7,8]. Diagnostic core biopsies stained with hematoxylin and eosin were evaluated for stromal lymphocyte infiltration. Areas evaluated include central tumor stroma and stroma at the invasive margin. Tumor cell areas, areas of necrosis and scarring were not included. Lymphocytes and plasma cells were included while neutrophils were excluded. Cases were reported as "percent stromal lymphocytes".

A clinical chart review was performed on the neoadjuvant breast cancer cases and included demographic and clinical information: age at diagnosis, self-reported ethnicity/race, tumor subtype as well as treatment history: neoadjuvant/adjuvant therapies, including chemotherapy, HER2 directed therapy, endocrine therapy, surgery, radiation. Patient charts were also reviewed for recurrence of breast cancer and death.

This study was reviewed and approved by the Hawaii Pacific Health and Queens Medical Center Institutional Review Boards for human subjects research. Statistical analysis was performed using the SAS 9.4 (SAS Institute Inc., Cary, NC) software. The REG procedure applied linear regression analyses on the continuous TILs (percentage tumor infiltrating lymphocytes) outcome. The predictor variables were race, age, subtype and health system. For the HPH subset analysis the variables included were: race, age, $\mathrm{BMI}$, stage, chemotherapy, subtype and PCR. We ran both univariable (unadjusted) and multivariable (adjusted for other predictors) models.

\section{Results}


A total of 183 cases of early stage breast cancer from patients who received neoadjuvant treatment were evaluated. The most commonly represented ethnicity was Asian with 72 cases (39.3\%), followed by Native Hawaiian/Pacific Islander (NHPI) with 53 cases (29\%), White with 48 cases (26.2\%), and Other with 10 cases (5.5\%) (Table1). The most common subtype was ER-/PR-/HER2- with 62 cases (33.9\%), followed by ER-/PR-/HER2 + with 56 cases (30.6\%), ER+ or PR+/HER2+ with 50 cases (27.3\%), and ER+ or $\mathrm{PR}+/ \mathrm{HER} 2-$ with 15 cases $(8.2 \%)$. The ethnicity with the most predominant proportion of cases with a pCR was Other with 5 cases (50.0\%), followed by NHPI with 26 cases (49.1\%), Asian with 29 cases (40.3\%), and White with 18 cases (37.5\%) (Table 1$)$.

Table 1 pCR according to Patient Race, Age and Subtype

\begin{tabular}{llll} 
Variable & & N (\%) & PCR N (\%) \\
\hline Race & Asian & $72(39.3)$ & $29(40.3)$ \\
\hline & NHPI & $53(29)$ & $26(49.1)$ \\
\hline & White & $48(26.2)$ & $18(37.5)$ \\
\hline Age & Other & $10(5.5)$ & $5(50)$ \\
\hline & $30-39$ & $19(10.4)$ & $12(63.1)$ \\
\hline & $40-49$ & $34(18.6)$ & $13(38.2)$ \\
\hline & $50-59$ & $46(25.1)$ & $23(50)$ \\
\hline \multirow{2}{*}{ Subtype } & ER-/PR-/HER2- & $62(33.9)$ & $19(30.6)$ \\
\hline & ER+ or PR+/HER2- & $15(8.2)$ & $2(13.3)$ \\
\hline & ER-/PR-/HER2+ & $56(30.6)$ & $37(66.1)$ \\
\hline & ER+ or PR+/HER2+ & $50(27.3)$ & $20(40)$
\end{tabular}

The percentage of sTILs in breast cancer tumors of all patients based on age at diagnosis, race and breast cancer subtype were evaluated. On univariable analysis, we found significantly greater percentages of sTILs among women who identified as Asian, NHPI and Other $(33.4 \%, 33.7 \%$ and $40.5 \%$, $p=0.04, p=0.05$ and $p=0.04$ respectively) (Table 2). On multivariable analysis, this remained significant for Asian and $\mathrm{NHPI}$ only (37.7\% and $37.2 \%, p=0.01$ and $p=0.02$ respectively) (Table 2 ). On univariable analysis, significantly lesser percentages of sTILs were seen among women between the ages of 60-69 years, however on multivariable analysis there were no significant differences found according to patient age (Table 2). With both univariable and multivariable analysis, there were significantly greater percentages of sTILs found among women who had ER-/PR-/HER2+ or TN breast cancer subtypes (Table 2). 
Table 2 Percent sTILs for all breast cancer patients receiving neoadjuvant treatment

\begin{tabular}{|c|c|c|c|c|c|c|c|c|c|c|c|}
\hline \multirow[b]{2}{*}{ Variable } & & \multirow[b]{2}{*}{$\mathrm{N}$} & \multirow[b]{2}{*}{$\%$} & \multicolumn{4}{|c|}{ UNIVARIABLE } & \multicolumn{4}{|c|}{ MULTIVARIABLE } \\
\hline & & & & Mean & $\mathrm{LCL}$ & $\mathrm{UCL}$ & $\mathrm{P}$ & Mean & $\mathrm{LCL}$ & $\mathrm{UCL}$ & $\mathrm{P}$ \\
\hline \multirow[t]{3}{*}{ Race } & Asian & 72 & 39.3 & 33.4 & 28.0 & 39.3 & .04 & 33.6 & 28.2 & 39.5 & .01 \\
\hline & NHPI & 53 & 29.0 & 33.7 & 27.4 & 40.6 & .05 & 34.3 & 28.0 & 41.2 & .01 \\
\hline & Other & 10 & 5.5 & 40.5 & 26.5 & 56.2 & .04 & 35.1 & 22.3 & 50.4 & .08 \\
\hline \multirow[t]{4}{*}{ Age } & $30-39$ & 19 & 10.4 & 41.1 & 30.5 & 52.5 & & 35.6 & 25.1 & 47.8 & \\
\hline & $40-49$ & 34 & 18.6 & 32.0 & 24.3 & 40.8 & .20 & 33.5 & 25.6 & 42.4 & .76 \\
\hline & $60-69$ & 53 & 29.0 & 28.1 & 22.0 & 35.3 & .05 & 27.7 & 21.7 & 34.6 & .22 \\
\hline & $70+$ & 31 & 16.9 & 27.1 & 19.3 & 36.7 & .05 & 26.0 & 18.7 & 34.9 & .17 \\
\hline \multirow[t]{4}{*}{ Subtype } & ER-/PR-/HER2- & 62 & 33.9 & 34.8 & 29.0 & 41.1 & & 35.3 & 29.3 & 41.7 & \\
\hline & $\mathrm{ER}+$ or PR+/HER2- & 15 & 8.2 & 25.0 & 14.7 & 39.2 & .20 & 19.4 & 10.5 & 33.3 & .04 \\
\hline & ER-/PR-/HER2+ & 56 & 30.6 & 36.3 & 30.2 & 42.9 & .73 & 35.7 & 29.6 & 42.3 & .92 \\
\hline & $\mathrm{ER}+$ or $\mathrm{PR}+/ \mathrm{HER} 2+$ & 50 & 27.3 & 23.3 & 17.2 & 30.8 & .02 & 23.7 & 17.8 & 30.9 & .02 \\
\hline
\end{tabular}

We evaluated additional variables from 79 cases including: $\mathrm{BMl}$ at diagnosis, stage, treatment regimen and pCR (Table 3). On univariable analysis, patients who obtained a pCR had a significantly higher sTIL percent $(40.6 \%$ vs $28.6 \%, p=0.04)$ but this was not found on multivariable analysis (Table 3$)$. Additionally, there were statistically significant race/ethnic differences in STILs on both univariable and multivariable analysis where NHPI and Asian patients had significantly greater percentages of sTILs in their tumors compared to White patients ( $38.2 \%, 34.3 \%$ vs $20.7 \%, p=0.01$ and $p=0.05$ respectively) (Table 3 ).

Table 3 Percent sTILs on subset of neoadjuvant cases with additional variables

\begin{tabular}{|c|c|c|c|c|c|c|c|c|c|c|c|}
\hline \multirow[b]{2}{*}{ Variable } & & \multirow[b]{2}{*}{$\mathrm{N}$} & \multirow[b]{2}{*}{$\%$} & \multicolumn{4}{|c|}{ UNIVARIABLE } & \multicolumn{4}{|c|}{ MULTIVARIABLE } \\
\hline & & & & Mean & $\mathrm{LCL}$ & $\mathrm{UCL}$ & $\mathrm{P}$ & Mean & $\mathrm{LCL}$ & $\mathrm{UCL}$ & $\mathrm{P}$ \\
\hline \multirow[t]{4}{*}{ Race } & Asian & 22 & 27.8 & 33.9 & 24.4 & 44.8 & .20 & 34.3 & 24.7 & 45.3 & .05 \\
\hline & NHPI & 25 & 31.6 & 41.0 & 31.8 & 50.9 & .02 & 38.2 & 28.1 & 49.5 & .01 \\
\hline & White & 25 & 31.6 & 24.4 & 16.1 & 35.4 & & 20.7 & 13.3 & 30.7 & \\
\hline & Other & 7 & 8.9 & 44.3 & 27.4 & 62.6 & .05 & 39.5 & 22.1 & 60.0 & .06 \\
\hline \multirow[t]{5}{*}{ Age } & $30-39$ & 10 & 12.7 & 35.0 & 21.5 & 51.4 & & 27.4 & 14.3 & 46.0 & \\
\hline & $40-49$ & 14 & 17.7 & 40.0 & 28.0 & 53.4 & .63 & 50.1 & 35.3 & 64.9 & .06 \\
\hline & $50-59$ & 25 & 31.6 & 40.0 & 30.8 & 50.0 & .59 & 37.0 & 28.1 & 46.9 & .32 \\
\hline & $60-69$ & 21 & 26.6 & 22.4 & 13.6 & 34.6 & .18 & 19.4 & 11.5 & 30.9 & .39 \\
\hline & $70+$ & 9 & 11.4 & 34.4 & 20.4 & 51.8 & .96 & 24.9 & 13.6 & 40.9 & .82 \\
\hline \multirow[t]{3}{*}{ BMI } & $<25$ & 38 & 48.1 & 31.7 & 24.2 & 40.4 & & 28.3 & 20.7 & 37.3 & \\
\hline & $25-30$ & 17 & 21.5 & 35.0 & 23.9 & 47.9 & .66 & 30.7 & 20.1 & 43.8 & .75 \\
\hline & $>30$ & 24 & 30.4 & 37.1 & 27.5 & 47.8 & .42 & 36.1 & 26.6 & 46.9 & .25 \\
\hline \multirow[t]{6}{*}{ Stage } & $1 \mathrm{~A}$ & 9 & 11.4 & 30.6 & 16.9 & 48.8 & & 23.8 & 12.0 & 41.7 & \\
\hline & $1 \mathrm{~B}$ & 13 & 16.5 & 31.9 & 20.0 & 46.8 & .90 & 28.1 & 16.4 & 43.9 & .69 \\
\hline & $2 \mathrm{~A}$ & 23 & 29.1 & 39.2 & 29.5 & 49.8 & .40 & 37.9 & 27.0 & 50.2 & .15 \\
\hline & $2 B$ & 17 & 21.5 & 38.2 & 27.2 & 50.7 & .47 & 37.9 & 25.5 & 52.1 & .21 \\
\hline & $3 \mathrm{~A}$ & 7 & 8.9 & 19.3 & 6.7 & 44.1 & .40 & 12.6 & 2.7 & 42.8 & .39 \\
\hline & $3 \mathrm{~B}$ & 10 & 12.7 & 31.5 & 18.3 & 48.6 & .93 & 33.4 & 18.7 & 52.3 & .44 \\
\hline \multirow[t]{5}{*}{ Treatment } & ACT & 22 & 27.8 & 33.0 & 23.3 & 44.3 & .66 & 41.1 & 23.7 & 60.9 & .39 \\
\hline & Letrozole & 4 & 5.1 & 16.2 & 3.0 & 54.7 & .26 & 14.6 & 3.0 & 48.3 & .06 \\
\hline & $\mathrm{TC}$ & 15 & 19.0 & 36.7 & 25.0 & 50.2 & & 50.6 & 34.2 & 66.9 & \\
\hline & $\mathrm{TCHP}$ & 25 & 31.6 & 34.4 & 25.3 & 45.0 & .79 & 21.3 & 11.1 & 37.0 & .03 \\
\hline & THP & 13 & 16.5 & 37.7 & 25.1 & 52.1 & .92 & 23.2 & 11.9 & 40.4 & .05 \\
\hline \multirow[t]{4}{*}{ Subtype } & ER-/PR-/HER2- & 21 & 26.6 & 36.4 & 26.5 & 47.7 & & 24.9 & 11.9 & 44.8 & \\
\hline & $\mathrm{ER}+$ or $\mathrm{PR}+/ \mathrm{HER} 2-$ & 15 & 19.0 & 25.0 & 14.4 & 39.7 & .20 & 19.0 & 8.9 & 36.0 & .51 \\
\hline & ER-/PR-/HER2+ & 28 & 35.4 & 39.5 & 30.6 & 49.1 & .67 & 44.5 & 29.2 & 61.0 & .21 \\
\hline & $\mathrm{ER}+$ or $\mathrm{PR}+/ \mathrm{HER} 2+$ & 15 & 19.0 & 29.7 & 18.6 & 43.7 & .43 & 31.3 & 18.1 & 48.4 & .64 \\
\hline \multirow[t]{2}{*}{$\mathrm{pCR}$} & no & 43 & 54.4 & 28.6 & 21.7 & 36.7 & & 28.6 & 21.0 & 37.7 & \\
\hline & yes & 36 & 45.6 & 40.6 & 32.7 & 48.9 & .04 & 34.2 & 26.1 & 43.4 & .39 \\
\hline
\end{tabular}


Percentage of pCR in breast cancer tumors of all patients based on age at diagnosis, race, and breast cancer subtype were evaluated (Table 4). In both univariable and multivariable analyses, we did not see significant differences in PCR among racial/ethnic groups studied (Asian, NHPI and Other) compared to White women. On univariable analysis, there was a significantly less likelihood of a pCR among women between the ages of 60-69 years compared to other age groups; however there were no significant differences found among all ages on multivariable analysis. In both univariable and multivariable, there was a significantly higher likelihood of obtaining a pCR among women who had ER-/PR-/HER2+ breast cancer (3.02 and 3.06 vs 0.6 and $0.59, p=0.01$ and $p=0.02$ respectively) compared to ER+/PR+/HER2breast cancer (Table 4).

Table 4 Pathologic complete response $(\mathrm{pCR})$ for all breast cancer patients

\begin{tabular}{|c|c|c|c|c|c|c|c|c|c|c|c|}
\hline \multirow[b]{2}{*}{ Variable } & & \multirow[b]{2}{*}{$\mathrm{N}$} & \multirow[b]{2}{*}{$\%$} & \multicolumn{4}{|c|}{ UNIVARIABLE } & \multicolumn{4}{|c|}{ MULTIVARIABLE } \\
\hline & & & & Mean & $\mathrm{LCL}$ & $\mathrm{UCL}$ & $\mathrm{P}$ & Mean & $\mathrm{LCL}$ & $\mathrm{UCL}$ & P \\
\hline \multirow[t]{4}{*}{ Race } & Asian & 72 & 39.3 & 33.4 & 28.0 & 39.3 & .04 & 33.6 & 28.2 & 39.5 & .01 \\
\hline & NHPI & 53 & 29.0 & 33.7 & 27.4 & 40.6 & .05 & 34.3 & 28.0 & 41.2 & .01 \\
\hline & White & 48 & 26.2 & 23.6 & 17.3 & 31.3 & & 22.0 & 16.1 & 29.4 & \\
\hline & Other & 10 & 5.5 & 40.5 & 26.5 & 56.2 & .04 & 35.1 & 22.3 & 50.4 & .08 \\
\hline \multirow[t]{5}{*}{ Age } & $30-39$ & 19 & 10.4 & 41.1 & 30.5 & 52.5 & & 35.6 & 25.1 & 47.8 & \\
\hline & $40-49$ & 34 & 18.6 & 32.0 & 24.3 & 40.8 & .20 & 33.5 & 25.6 & 42.4 & .76 \\
\hline & $50-59$ & 46 & 25.1 & 33.3 & 26.6 & 40.8 & .25 & 33.0 & 26.5 & 40.4 & .70 \\
\hline & $60-69$ & 53 & 29.0 & 28.1 & 22.0 & 35.3 & .05 & 27.7 & 21.7 & 34.6 & .22 \\
\hline & $70+$ & 31 & 16.9 & 27.1 & 19.3 & 36.7 & .05 & 26.0 & 18.7 & 34.9 & .17 \\
\hline \multirow[t]{4}{*}{ Subtype } & ER-/PR-/HER2- & 62 & 33.9 & 34.8 & 29.0 & 41.1 & & 35.3 & 29.3 & 41.7 & \\
\hline & ER+ or PR+/HER2- & 15 & 8.2 & 25.0 & 14.7 & 39.2 & .20 & 19.4 & 10.5 & 33.3 & .04 \\
\hline & ER-/PR-/HER2+ & 56 & 30.6 & 36.3 & 30.2 & 42.9 & .73 & 35.7 & 29.6 & 42.3 & .92 \\
\hline & $\mathrm{ER}+$ or $\mathrm{PR}+/ \mathrm{HER} 2+$ & 50 & 27.3 & 23.3 & 17.2 & 30.8 & .02 & 23.7 & 17.8 & 30.9 & .02 \\
\hline
\end{tabular}

\section{Discussion}

Our findings showed significantly higher percentages of sTILs in tumors of Asians and NHPIs compared to those of White breast cancer patients. We however did not see any significant difference in PCR among these racial/ethnic groups. This study addresses a gap in the literature by examining differences in sTILs among understudied racial/ethnic groups, particularly Native Hawaiian/Pacific Islander (NHPI) and Asian women. These findings expand upon a subset of this data that was previously reported by our group showing significantly higher percentages of sTILs in Asian, NHPI, and Other women compared to White women [9]. Chen et al. showed Asian patients were found to have significantly higher amounts of tumorinfiltrating immune cells compared to White patients, particularly CD4+ T cells and in Luminal $B$ and HER2+ tumors [6]. Our results may suggest differences in immune cell profiles of TILs in Asians and NHPI tumors that may vary from those in Whites, which should be further evaluated and characterized. Jiang et al. showed significantly higher overall survival in patients with breast cancer tumors enriched with specific TILs including: B cells, NK cells, CD8+ T cells, CD4+ activated T cells, and low yס T cells, neutrophils, and activated mast cells [10]. Additional data show that TIL immune profiles high in M2 macrophages may also be associated with poorer clinical outcomes while those high in CD4+ Th1 helper T cells may be more strongly associated with improved survival $[6,11]$. Higher percentages of sTILs in 
tumors of Asians and NHPI may reflect increased tumor infiltration of immune cells that have weaker associations with immunological response, $\mathrm{pCR}$, and patient clinical outcomes/prognosis.

Current studies regarding differences in TILs and their contributions to patient prognosis among different racial/ethnic groups have shown inconsistent results. O'Meara et. al. showed that TILs amount and immune gene expressions are not significantly different between Whites and Blacks with TN breast cancer [12]. In contrast, Abdou et al. found breast cancer tumors from Black patients had higher CD8+ T cell tumor infiltrating densities than in White patients [4]. In previous studies, Black breast cancer patients had a higher amount of macrophage infiltrating tumors with a high number of CD163+ macrophages and increased macrophage proliferation [13]. Carrio et al. showed CD68+ macrophages were significantly more abundant in tumors from Black and Latina patients but showed no differences in overall survival when compared to White patients [14]. Another study found higher levels of Proliferating Cell Nuclear Antigen positive (PCNA+) tumor-associated macrophages and shorter disease-free survival in Latina patients compared to White patients [15]. Given the poor prognosis seen among the NHPI breast cancer patients [16], characterizing these TILs is an important future step to better understand how the tumor microenvironment may contribute to these disparities.

Some studies have also noticed differences in TIL amount when stratified for variables other than patient race. In Black women, $\mathrm{CD} 8+\mathrm{T}$ cell density was seen to be higher in patients who were younger or with hormone-negative high grade tumors [4]. However, in western Kenyan patients, immune cell infiltration density was significantly associated with increased tumor proliferation index but not significantly correlated with molecular subtype or tumor grade [17]. In another study, no differences in STIL percentages were observed to be associated with patient age or BMI at diagnosis or tumor stage [9].

Similarly, the literature shows inconsistencies with regards to relationships between TIL count/density and grade/stage of breast tumors across racial groups. O'Meara et al. showed no significant difference in TIL amount between Black and White breast cancer patients [12]. However, Abdou et al. showed higher TILs amount can be found in tumors of younger patients, hormone-negative tumors, and high grade tumors in both Black and White patients, irrespective of race [4].

We saw significantly lower rates of PCR among women between the ages of 60-69 years compared to other age groups. Although we did not see differences in sTILs according to age in our study, prior data show lower percentages of sTILs among women in this patient age. There are known lower TIL percentages in tumors of older patients [4]. Our findings support data showing older cancer patients receiving treatment are less likely to have a pathologic complete response irrespective of race/ethnicity. In our subset of patients being analyzed for additional variables, we did not see any differences in sTILs according to $\mathrm{BMI}$ at diagnosis, stage or treatment regimen.

Limitations to this study include incomplete patient medical records with variable neoadjuvant treatment data. Although we were able to obtain a subset of cases with complete medical data, we did not see differences when accounting for those additional variables. Although the pathologic TILs evaluations were performed according to the International Immuno-Oncology Biomarker Working Group, the cases 
were evaluated by two sets of different pathologists, introducing possible variability. This approach however, provides an avenue to broadly incorporate TILs measurement in pathology reports. Our medical center has already routinely reported on sTILs percent and volume for all neoadjuvant TN and HER2+ breast cancer cases. Finally, neoadjuvant cases prior to 2015 are sparse as TN and HER2+ historic treatment strategies did not routinely focus on the neoadjuvant approach.

\section{Conclusions}

Racial/ethnic differences in the amount of sTILs in breast cancer tumors suggest that higher STIL percentages alone do not predict for pCR. Increases in sTILs in Asian and NHPI patients suggest differences in immune cell profiles in the breast tumor microenvironment. This may in part contribute to known outcome disparities in these populations.

\section{Declarations}

\section{Author's contributions}

All authors made substantial contributions to conception and design, analysis and interpretation of data, and critical review of the manuscript. JF conceived of the study, coordinated the data acquisition and analysis, generated tables and figures and helped to draft the manuscript. IP contributed to the statistical methods, data analysis, generated tables and figures and helped to draft the manuscript. AS and SB contributed to the data analysis and helped to draft the manuscript. AT, KV, CW and MF contributed to the data acquisition and analysis and helped to draft the manuscript. All authors read and approved the final manuscript.

\section{Funding}

This work was completed as part of the funding from the University of Hawaii Cancer Center P30 CA071789 Grant from the National Institutes of Health (J. Fukui, I. Pagano). The funding body had no role in the design; in the collection, analysis, or interpretation of data; in writing of the manuscript; or in the decision to submit the manuscript for publication.

\section{Conflicts of interest/Competing interest}

The authors declare that they have no competing interests.

\section{Ethics approval and Consent to participate}

The Institutional Review Boards at the University of Hawaii, Hawaii Pacific Health and Queens Medical Center approved this study. All aspects of the investigation compiled with current rules for research involving Human Subjects.

\section{Availability of data and material}


The datasets used and/or analyzed during the current study are available from the corresponding author on reasonable request.

\section{Acknowledgements}

The authors would like to thank Hawaii Pacific Health and the Queens Medical Center for its collaboration efforts and to the University of Hawaii Cancer Center for its financial support of this work.

\section{References}

1. Basu A, Ramamoorthi G, Jia Y, et al. Chapter Six-Immunotherapy in breast cancer: Current status and future directions. Advances in Cancer research. 2019;143:295-349.

2. Loi S, Michiels S, Salgado R, et al. Tumor infiltrating lymphocytes are prognostic in triple negative breast cancer and predictive for trastuzumab benefit in early breast cancer: results from the FinHER trial. Ann Oncol. 2014;25(8):1544-1550. doi:10.1093/annonc/mdu112

3. Seo AN, Lee HJ, Kim EJ, et al. Tumour-infiltrating CD8+ lymphocytes as an independent predictive factor for pathological complete response to primary systemic therapy in breast cancer. $\mathrm{Br} \mathrm{J}$ Cancer. 2013;109:2705-13. doi: 10.1038/bjc.2013.634.

4. Abdou Y, Attwood K, Cheng TD, et al. Racial differences in CD8+ T cell infiltration in breast tumors from Black and White women. Breast Cancer Res. 2020;22(1):62. doi:10.1186/s13058-020-01297-4.

5. Ali HR, Provenzano E, Dawson SJ, et al. Association between CD8+ T-cell infiltration and breast cancer survival in 12,439 patients. Ann Oncol. 2014;25: 1536-43. doi: 10.1093/annonc/mdu191.

6. Chen CH, Lu YS, Cheng AL, et al. Disparity in Tumor Immune Microenvironment of Breast Cancer and Prognostic Impact: Asian Versus Western Populations. Oncologist, 2020;25(1):e16-e23. doi:10.1634/theoncologist.2019-0123.

7. Hendry S, Salgado R, Gevaert T, et al. Assessing tumor-infiltrating lymphocytes in solid tumors: A practical review for pathologists and proposal for a standardized method from the international immunooncology biomarkers working group: Part 1: assessing the host immune Response, TILs in invasive breast carcinoma and ductal carcinoma in situ, metastatic tumor deposits and areas for further research. Adv Anat Pathol. 2017;24:235-251. doi: 10.1097/PAP.0000000000000162.

8. Salgado R, Denkert C, Demaria, S, et al. The evaluation of tumor-infiltrating lymphocytes (TILS) in breast cancer: Recommendations by an International TILs Working Group 2014. Ann Oncol. 2015;26:259-271. doi: 10.1093/annonc/mdu450.

9. Fukui J, Taniguchi A, Meister M, Pagano I, Killeen J. Racial/ethnic differences in tumor infiltrating lymphocytes [abstract]. In: Proceedings of the 2019 San Antonio Breast Cancer Symposium; 2019 Dec 10-14; San Antonio, TX. Philadelphia (PA): AACR; Cancer Res 2020;80(4 Suppl): Abstract nr P606-13. https://cancerres.aacrjournals.org/content/80/4_Supplement/P6-06-13

10. Jiang J, Pan W, Xu Y, et al. Tumour-Infiltrating Immune Cell-Based Subtyping and Signature Gene Analysis in Breast Cancer BAsed on Gene Expression Profiles. J Cancer. 2020;11(6):1568-1583. 
Published 2020 Jan 14. doi:10.7150/jca.37637.

11. Sawe RT, Kerper M, Badve S, et al. Aggressive breast cancer in western Kenya has early onset, high proliferation, and immune cell infiltration. BMC Cancer Res Treat. 2016; 158(1): 113-126.

12. O'Meara T, Safonov A, Casadevall D, et al. Immune microenvironment of triple-negative breast cancer in African-American and white women. Breast Cancer Res Treat. 2019;175:247-59. doi: 10.1007/s10549-019-05156-5.

13. Koru-Sengul T, Santander AM, Miao F, et al. Breast cancers from black women exhibit higher numbers of immunosuppressive macrophages with proliferative activity and of crown-like structures associated with lower survival compared to non-black Latinas and Caucasians. Breast Cancer Res Treat. 2016;158:113-26. doi: 10.1007/s10549-016-3847-3.

14. Carrio R, Koru-Sengul T, Miao F, et al. Macrophages as independent prognostic factors in small T1 breast cancers. Oncol Rep. 2013;29:141-8. doi.org/10.3892/or.2012.2088.

15. Mukhtar RA, Moore AP, Nseyo 0 , et al. Elevated PCNA+ tumor-associated macrophages in breast cancer are associated with early recurrence and non-white ethnicity. Breast Cancer Res Treat. 2011;130:635-44. doi: 10.1007/s10549-011-1646-4.

16. Loo LWM, Williams M, Hernandez BY. The high and heterogeneous burden of breast cancer in Hawaii: A unique multiethnic U.S. Population. Cancer Epidemiol. 2019;58:71-76. doi:10.1016/j.canep.2018.11.006.

17. Sawe RT, Mining SK, Ofulla AV, et al. Tumor infiltrating leukocyte density is independent of tumor grade and molecular subtype in aggressive breast cancer of Western Kenya. Trop Med Health. 2017;45:19. Published 2017 Aug 4. doi:10.1186/s41182-017-0059-4. 